\title{
Combined minimally invasive management of infected pancreatic necrosis: a case report
}

\author{
Łukasz Wysocki, Marek Wroński, Włodzimierz Cebulski, Ireneusz Wojciech Krasnodębski \\ Department of General, Gastroenterological and Oncological Surgery, Medical University of Warsaw, Poland
}

Videosurgery Miniinv (e-pub, ahead of print) DOI: 10.5114/wiitm.2014.40989

\begin{abstract}
Infected necrosis is a potentially fatal complication of necrotizing pancreatitis. Open surgical debridement is the mainstay management of infected pancreatic necrosis. Over the last decade minimally invasive techniques have been increasingly used for the treatment of infected pancreatic necrosis and their results are encouraging. However, the optimal technique of minimal access necrosectomy and the timing of intervention have not been established yet. Patients with septic complications of acute pancreatitis represent a challenging group which requires individualized management often involving numerous techniques. We report a case of a 52-year-old patient in whom 3 minimally invasive techniques were needed for complete recovery.
\end{abstract}

Key words: acute pancreatitis, pancreatic necrosectomy, infected pancreatic necrosis, laparoscopy, endoscopic retrograde cholangiopancreatography.

\section{Introduction}

Infected pancreatic necrosis (IPN) is the most threatening complication of severe acute pancreatitis. Open surgical necrosectomy is still the procedure of choice in the treatment of infected pancreatic necrosis and debridement is usually performed through laparotomy [1]. Nevertheless, surgical treatment results in high rates of morbidity (34\% to 95\%) and mortality (11\% to 39\%) [2-4]. Recently, several techniques of minimal access pancreatic necrosectomy have been reported [5-8]. Modern management of necrotizing pancreatitis involves usage of multiple minimally invasive techniques usually at different stages of the disease.

We report a case of a patient who initially underwent percutaneous catheter drainage of infected pancreatic necrosis with subsequent retroperitoneoscopic minimal access necrosectomy for persistent local sepsis. Postnecrosectomy pancreatic fistula was successfully managed by endoscopic stenting of the main pancreatic duct.

\section{Case report}

A 52-year-old woman was referred to our department from a district hospital for further treatment of acute pancreatitis. The etiology of acute pancreatitis was idiopathic. The initial Computed Tomography Severity Index of acute pancreatitis (CTSI) was 7 points. The patient's past medical history was significant for insulin-dependent diabetes mellitus, dyslipidemia and sigmoidectomy for colorectal adenocarcinoma 5 years earlier. Computed tomography scans revealed a diffuse acute necrotic collection (ANC) involving the body and tail of the pancreas which extended anterior to the left kidney. The patient received maximal conservative treatment including intensive fluid replacement, enteral and parenteral nutrition. The patient's clinical condition

\section{Address for correspondence}

Łukasz Wysocki MD, PhD, Department of General, Gastroenterological and Oncological Surgery, Medical University of Warsaw,

1 A Banacha St, 02-097 Warsaw, Poland, phone: +48 604433 095, e-mail: wysocki@adres.pl 
deteriorated in the fourth week of the disease with fever and increased serum C-reactive protein (CRP) of $432 \mathrm{mg} / \mathrm{l}$. The antibiotic therapy was modified and piperacillin with tazobactam was started. She improved and was discharged asymptomatic.

The patient was rehospitalized 10 weeks later because of infected pancreatic necrosis. On admission, there was a tender mass in the left epigastrium. Laboratory investigations showed CRP of $239 \mathrm{mg} / \mathrm{l}$. This time computed tomography revealed a walled-off necrosis (WON) with gas bubbles (Figure 1). The lesion was located in the lesser sac and extended through the left retrocolic region down to the pelvis. The patient underwent ultrasound-guided percutaneous catheter drainage of this fluid collection and a $32 \mathrm{Fr}$ drain was inserted through the left retroperitoneal access situated between the descending colon and the left kidney. Purulent fluid with necrotic debris was drained. Culture of the fluid grew Escherichia coli, Bacteroides fragilis and Peptostreptococcus spp. Because of persistent local sepsis despite 2 weeks' percutaneous drainage, we decided to perform a percutaneous retroperitoneal pancreatic necrosectomy using a single-access port SILS (Covidien Poland). This is a special port made of a flexible soft foam with access channels for 3 trocars. This technique is fully described elsewhere [9]. In short, the lumbar incision was dilated and a single-access port was placed. The necrotic debris was removed piecemeal using grasping forceps under the visual guidance of a laparoscope. The postnecrotic

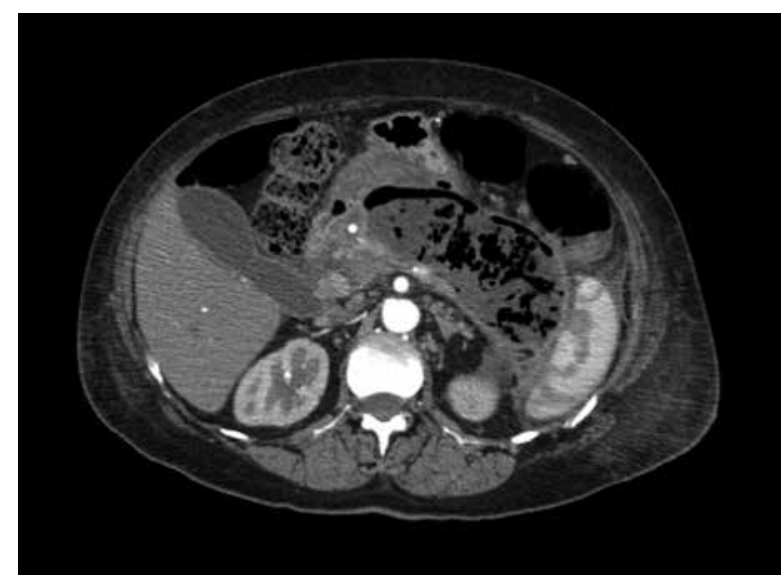

Figure 1. Computed tomography scan shows infected walled-off necrosis (WON) located within the lesser sac and extending anterior to the left kidney cavity was thoroughly irrigated and a large-bore drain was left for gravitational drainage. Postoperatively, the patient's condition improved and the inflammatory parameters normalized. There was drain discharge of 300-400 $\mathrm{ml}$ per day with a high amylase content $(62684 \mathrm{U} / \mathrm{l})$. Two weeks after the necrosectomy, endoscopic retrograde cholangiopancreatography (ERCP) was performed and showed leakage from the main pancreatic duct in the proximal part of the pancreatic body (Figure 2). Sphincterotomy was done and a short, transpapillary $7 \mathrm{Fr}$ stent was inserted. Drain discharge decreased gradually within the next 2 weeks and it was removed. The patient was discharged on the $49^{\text {th }}$ day after the initial percutaneous drainage and she remains asymptomatic a year after discharge.

\section{Discussion}

Pancreatic necrosis becomes infected in 40-70\% of patients [1]. Current data favor a so-called step-up approach in the management of IPN that involves less invasive procedures at the beginning of treatment, whereas more aggressive interventions are used if the former fail [10]. Our patient responded well to systemic antibiotics administered for the first epi-

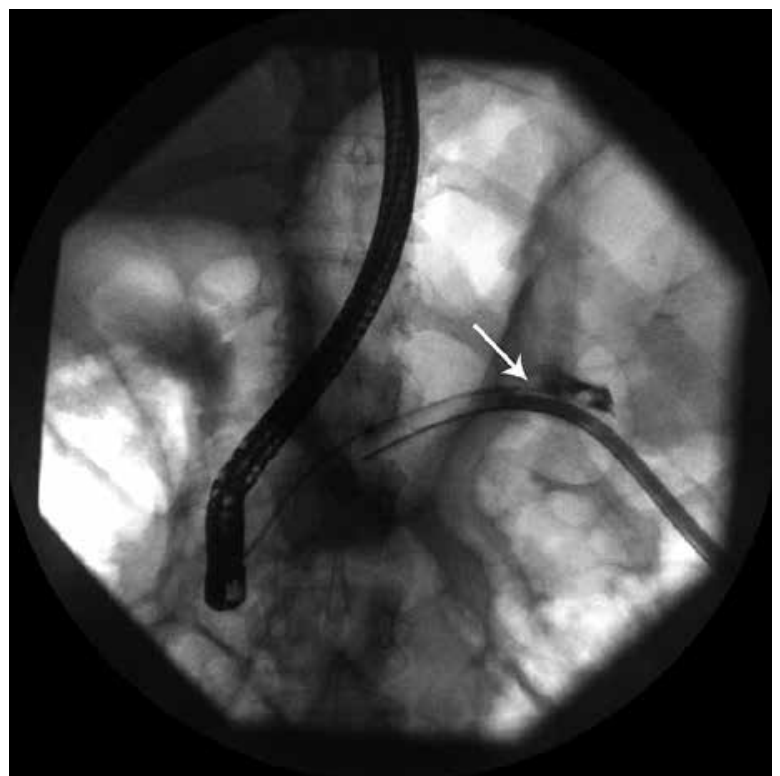

Figure 2. Endoscopic retrograde cholangiopancreatography ductal disruption in the body of the pancreas (arrow) - contrast outflow into the postnecrotic cavity and abdominal drain 
sode of infected pancreatic necrosis. Similarly, recent evidence supports initial conservative therapy with susceptibility-guided antibiotics in patients with IPN who remain in good clinical condition [11]. Patients unresponsive to conservative therapy should undergo percutaneous catheter drainage or endoscopic drainage as the next step. Failure of either of these treatment modalities requires formal pancreatic necrosectomy which can be done through the retroperitoneal minimal access or endoscopically. Open necrosectomy is recommended in patients in whom minimally invasive treatment is not feasible or unsuccessful. Our patient underwent percutaneous catheter drainage for infected walled-off necrosis. Although a large-bore drain was inserted already at the initial drainage procedure, it failed to evacuate the particulate debris, which resulted in persistent local sepsis. Retroperitoneoscopic minimal access necrosectomy allowed almost complete removal of the necrotic debris and controlled infection in this patient. Several techniques of minimally invasive pancreatic necrosectomy have been developed [3, 5-8]. Percutaneous retroperitoneal pancreatic necrosectomy is best suited for patients with necrosis and fluid collections extending into the pararenal spaces, especially on the left side.

One of the most common complications of percutaneous drainage or necrosectomy is pancreatic fistula. The incidence of pancreatic fistula is varied and ranges from $3 \%$ to $72 \%$ [3]. Pancreatic fistula after necrosectomy resolves spontaneously in approximately $80 \%$ of patients; however, it often takes more than 6 months to heal the fistula [12]. Endoscopic stenting of the pancreatic duct enhances fistula closure by both decreasing intraductal pressure and mechanical coverage at the site of ductal disruption. However, the optimal timing of endoscopic intervention is controversial. In our patient, the pancreatic fistula healed within 2 weeks after the endoscopic treatment, although the stent was placed only across the papilla and did not reach the site of leakage.

In conclusion, modern management of infected pancreatic necrosis often requires the use of several minimally invasive techniques. In our patient, we successfully combined 3 such techniques: percutaneous catheter drainage, retroperitoneoscopic minimal access necrosectomy and endoscopic stenting of the main pancreatic duct, which resulted in her complete recovery.

\section{References}

1. Uhl W, Warshaw A, Imrie C, et al. IAP guidelines for the surgical management of acute pancreatitis. Pancreatology 2002; 2: 565 73.

2. Rau B, Bothe A, Beger HG. Surgical treatment of necrotizing pancreatitis by necrosectomy and closed lavage: changing patient characteristics and outcome in a 19-year, single-center series. Surgery 2005; 138: 28-39.

3. Connor S, Alexakis N, Raraty MG, et al. Early and late complications after pancreatic necrosectomy. Surgery 2005; 137: 499-505.

4. Howard TJ, Patel JB, Zyromski N, et al. Declining morbidity and mortality rates in the surgical management of pancreatic necrosis. J Gastrointest Surg 2007; 11: 43-9.

5. Horvath K, Freeny P, Escallon J, et al. Safety and efficacy of video-assisted retroperitoneal debridement for infected pancreatic collections: a multicenter, prospective, single-arm phase 2 study. Arch Surg 2010; 145: 817-25

6. Seifert H, Biermer M, Schmitt W, et al. Transluminal endoscopic necrosectomy after acute pancreatitis: a multicentre study with long-term follow-up (the GEPARD Study). Gut 2009; 58: 1260-6.

7. Connor S, Ghaneh P, Raraty M, et al. Minimally invasive retroperitoneal pancreatic necrosectomy. Dig Surg 2003; 20: 270-7.

8. Šileikis A, Beiša V, Beiša A, et al. Minimally invasive retroperitoneal necrosectomy in management of acute necrotizing pancreatitis. Videosurgery Miniinv 2013; 8: 29-35.

9. Wronski M, Cebulski W, Slodkowski M, et al. Retroperitoneal minimally invasive pancreatic necrosectomy using single-port access. Surg Laparosc Endosc Percutan Tech 2012; 22: e8-11.

10. van Santvoort HC, Besselink MG, Bakker OJ, et al. A step-up approach or open necrosectomy for necrotizing pancreatitis. N Engl J Med 2010; 362: 1491-502.

11. Runzi M, Niebel W, Goebell H, et al. Severe acute pancreatitis: nonsurgical treatment of infected necroses. Pancreas 2005; 30 : 195-9.

12. Boerma D, Rauws E A, van Gulik TM, et al. Endoscopic stent placement for pancreaticocutaneous fistula after surgical drainage of the pancreas. Br J Surg 2000; 87: 1506-9.

Received: 3.03.2013, accepted: 16.05.2013. 